\title{
The effect of single-nucleotide polymorphisms within heat shock protein beta 1 on beef quantity in Korean native steers
}

\author{
Jung-Keun Suh ${ }^{1,2}$, Jae-Sung Lee ${ }^{1}$, Hongsik Kong ${ }^{3}$, Yoonseok Lee ${ }^{3,4, \star}$, and Hong-Gu Lee ${ }^{1,2, \star}$ \\ ${ }^{1}$ Department of Animal Science and Technology, Sanghuh College of Life Sciences, \\ Konkuk University, Seoul, 05029, Republic of Korea \\ ${ }^{2}$ Team of An Educational Program for Specialists in Global Animal Science, Brain Korea 21 Plus Project, \\ Sanghuh College of Life Sciences, Konkuk University, Seoul, 05029, Republic of Korea \\ ${ }^{3}$ Department of Biotechnology, Hankyung National University, Anseong-si, \\ Gyeonggi-do, 17579, Republic of Korea \\ ${ }^{4}$ Center for Genetic Information, Hankyung National University, Anseong-si, \\ Gyeonggi-do, 17579, Republic of Korea \\ These authors contributed equally to this work.
}

Correspondence: Hong-gu Lee (hglee66@konkuk.ac.kr) and Yoonseok Lee (yoonseok95@hknu.ac.kr)

Received: 24 June 2019 - Revised: 24 May 2020 - Accepted: 24 June 2020 - Published: 17 November 2020

\begin{abstract}
Heat shock protein beta $1(H S P B 1)$, a member of the heat-shock family of protein, is a relatively small $(27 \mathrm{kDa})$ molecular chaperone protein associated with cellular development. The relationship between $H S P B 1$ expression and muscle growth in beef cattle has previously been reported, but there have been no reports of DNA markers related to meat quantity in Korean native steers. Therefore, the aim of this study was to evaluate the relationship of single-nucleotide polymorphisms (SNPs) within HSPBI in terms of the carcass traits related to muscle growth in Korean native steers. Through direct sequencing, we discovered three SNPs: g.111 T>C SNP (rs208395876) and g.2548 C> G SNP (rs483014585) were respectively located in 5' UTR (untranslated region) and $3^{\prime}$ UTR. Further, g.2352 T > C SNP (rs110832311) was located in the adjacent region of the RNA splicing site. The least square means of steers with a CC genotype of g.111 T $>$ C SNP had a significantly higher meat ratio $(P=0.04)$, while the least square means of steers with a CC genotype of g.2352 T $>C$ SNP had a significantly higher meat ratio $(P=0.002)$ and lower back-fat thickness $(P=0.004)$ than those of the other genotype. Moreover, although the least square means of steers with CC-CC, CT-CC, and TT-CC genotypes were significantly decreased for back-fat thickness, they were significantly increased for the meat ratio. Therefore, our results suggested that g. $111 \mathrm{~T}>\mathrm{C} \mathrm{SNP}$ and g.2352 T >C SNP could be a causal mutation related to an adipose metabolism in Korean cattle steer.
\end{abstract}

\section{Introduction}

Recently, several studies have evaluated the relationship between heat-shock protein beta $1(H S P B 1)$ and muscle growth (Dubińska-Magiera et al., 2014; Hamelin et al., 2006). $H S P B 1$ is a $27 \mathrm{kDa}$ small heat-shock protein that is expressed in many vertebrate tissues, particularly muscle. The general functions of $H S P B 1$ in muscle are to protect cells from physiological stress, inhibit cell death, and chaperone activity
(Arrigo, 2017). In previous studies, HSPBI has also been shown to be relevant in terms of feed efficiency (Jung et al., 2017). HSPB1 is also involved in muscle development in many species, including those reported in Dubińska-Magiera et al. (2014). HSPB 1 has been shown to be related to muscle hypertrophy in vivo (Hamelin et al., 2006). In a recent study, the expression of $H S P B I$ was found to be significantly increased during bovine myogenic differentiation compared to in the un-differentiation stage in myogenic cells, and it was 
also shown to be regulated by androgen-mediated myogenesis (Zhang et al., 2012, 2014).

In the beef industry, efficient muscle growth and development in cattle is critical for meat production (He et al., 2017).

Myogenesis is regulated by several myogenic genes, such as MyoD, Myogenin, MRF4, and Desmin (Charge and Rudnicki, 2004). These myogenic marker genes are known to be upregulated during myogenic differentiation (Sweetman, 2012). HSPB 1, a major factor of actin polymerization in muscle, is known to play a role in maintaining muscle structure (Sugiyama et al., 2000). HSPB1 also shows chaperone function, including preventing protein degradation, inhibiting muscle atrophy, and stabilizing muscle protein (Tucker and Shelden, 2009). It has been speculated that HSPBI enhances muscle development by protecting muscle proteins (Perng et al., 1999). In a recent study, the inhibition of $H S P B 1$ expression during myogenesis was shown to repress the expression of MyoD, Myogenin, and Desmin; formation of myotubes; and protein synthesis (Kim et al., 2018). A relationship has also been reported between HSPBI-10 expression and muscle growth in beef cattle, but there have been no reports of DNA markers related to meat quantity in Korean native steers. Therefore, the aim of this study was to evaluate the relationship of SNPs within HSPB1 with carcass traits related to muscle growth in Korean native steers.

\section{Materials and methods}

\subsection{Animals, DNA extraction, SNP discovery}

A total of 192 Korean cattle (body weight $\mathrm{BW}=464.68 \pm 45.09 \mathrm{~kg} ; 32.18$ months old [SD1.0]) raised in Pyeongchang (Gangwon, Republic of Korea) were used in this study. All of the steers were maintained under constant environmental conditions, such as having access to two types of commercial feeds at six feedlots. Muscle tissues were sampled from slaughtered individuals. Therefore, there is no certificate of animal ethics. Genomic DNA was extracted from longissimus dorsi muscle tissue using a LaboPass $^{\mathrm{TM}}$ tissue mini kit (Cosmo Genetech, Seoul, Korea). In order to discover SNPs, the bovine HSPBI sequence was obtained from the NCBI database (AC_000182.1). The primer sequence was designed using NCBI Premer-BLAST based on the selected polymorphism sites, and the primer information is shown in Table 1 . The sequencing was performed as outlined in a previous study (Lee et al., 2010), and SNPs were discovered using the Sequencer v5.2.4 program (Gene Codes Corp., Ann Arbor, MI). In order to map the functional SNPs on DNA, mRNA, and protein sequences, they were aligned using the Graphical View Legend on the NCBI database.

\subsection{SNP genotyping and statistical analysis}

Large-scale SNP genotyping was performed commercially using the Fluidigm ${ }^{\circledR}$ SNPTM Type assay as described in a previous study (Oh et al., 2018). In order to evaluate the association SNPs and carcass traits, the data were analyzed using the general linear model (GLM) of SPSS v. 22 (IBM, USA). The model is as follows:

$\mathrm{Yijkl}=\mu+\mathrm{Pi}+\mathrm{GSj}+\mathrm{SNPk}+\beta$ agel + eijkl,

where Yijkl is a phenotype of the carcass trait, $\mu$ is the overall mean for each trait, $\mathrm{Pi}$ is the feed type in farms, GSj is the random effect of the sire, SNPk is the fixed effect of SNP genotype, $\beta$ agelis the covariance of days bred, and eijkl is the random error.

\section{Results and discussion}

Bovine $H S P B 1$ has been shown to be closely associated with cell growth and bovine myogenesis in certain types of muscles. Thus, based on the results reported by Kim et al. (2018), we found that SNPs, which are directly regulated by gene expression, were discovered, and then we identified the relationship of their SNPs with beef quantity.

The position of SNP in DNA, RNA, and the protein sequence of the HSPBI gene as well as their alignments are shown in Fig. 1. In the present study, we discovered three SNPs using direct sequencing. These SNPs were located in $5^{\prime}$ UTR, intron 2, and $3^{\prime}$ UTR; g.111 T >C SNP (rs208395876) and g.2548 C > G SNP (rs483014585) are in 5' UTR and 3' UTR, while g.2352 T > C SNP (rs110832311) is in the region adjacent to the RNA splicing site. However, SNPs were not discovered in the exon region.

The multiple and single effects of SNPs within HSPBI on carcass traits, such as beef quantity, are shown in Tables 1 and 2. A previous study (unpublished data) demonstrated that the g.2548 C>G SNP (rs483014585) had no effects on carcass traits, and we thus removed the related data from the present study, as we could not use it as evidence to support our result. As shown in Table 1, g.111 T >C SNP was significantly associated with meat ratio $(P>0.05)$. The least square mean in the steer group with a $\mathrm{CC}$ genotype of g.111 T>C SNP was significantly higher than that in the steer group with other genotypes. Moreover, g.2352 T >C SNP was significantly associated with back-fat thickness and meat ratio $(P<0.01)$. Regarding back-fat thickness, the least square mean in the steer group with the CC genotype of g.2352 T > C SNP was significantly lower than that in the steer group with other genotypes. On the other hand, in terms of meat ratio, this group was significantly higher than that in the steer group with other genotypes.

As shown in Table 2, consistent with the results of a single effect, the combination genotype of g.111 T >C and g.2352 T $>$ C SNPs was significantly associated with back-fat thickness and meat ratio $(P<0.01)$. The steer group with combi- 


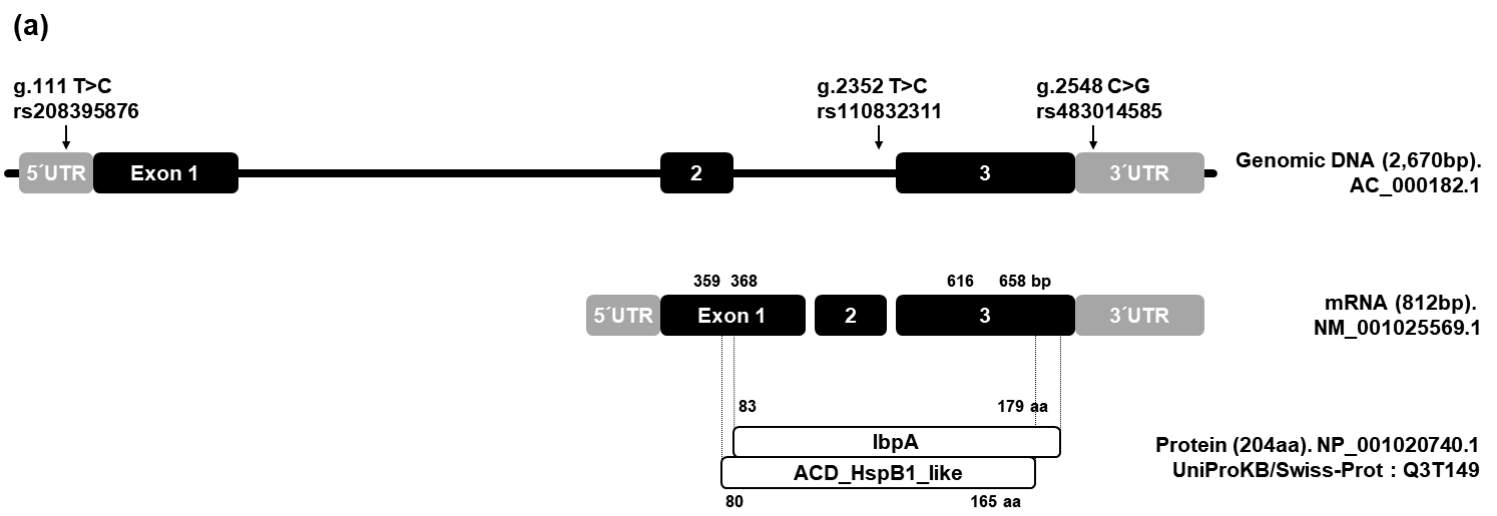

(b)

\begin{tabular}{|c|c|c|}
\hline \multirow{2}{*}{$\begin{array}{r}\text { Exon } 2 \\
\text { GCAAATACAC }\end{array}$} & \multicolumn{2}{|c|}{ Intron 2} \\
\hline & CGGATCT & GGCGCGCGG \\
\hline & Intron 2 & Exon 3 \\
\hline
\end{tabular}

Figure 1. Position of SNPs within the HSPB1 gene. (a) Black and grey boxes represent UTR and exon, respectively. (b) Position of g.2352 SNP $\left(^{*}\right)$ in RNA splicing site. Grey boxes represent exon 2 and exon 3 of HSPB1 gene. The italic letters represent the intron 2 region. Underscores refer to the donor and acceptor sites of the RNA splicing site.

Table 1. The single effect of g.111 T >C SNP and g.2352 T > C SNP within HSPB1 gene on carcass traits in a commercial Korean native steer population.

\begin{tabular}{|c|c|c|c|c|c|c|c|c|}
\hline \multirow{3}{*}{ Carcass traits } & \multicolumn{8}{|c|}{ Genotype (number of animals) } \\
\hline & \multicolumn{4}{|c|}{ g. $111 \mathrm{~T}>\mathrm{C} \mathrm{SNP}$} & \multicolumn{4}{|c|}{ g. $2352 \mathrm{~T}>\mathrm{C} \mathrm{SNP}$} \\
\hline & $\mathrm{CC}(6)$ & CT (66) & TT (118) & $p$ value & $\mathrm{CC}(42)$ & CT (91) & TT (57) & $p$ value \\
\hline Back-fat thickness, mm & $12.5 \pm 1.65$ & $13.15 \pm 0.51$ & $14.45 \pm 0.38$ & 0.084 & $12.09 \pm 0.63^{\mathrm{a}}$ & $14.48 \pm 0.42^{\mathrm{b}}$ & $14.31 \pm 0.53^{\mathrm{b}}$ & 0.004 \\
\hline Rib-eye area, $\mathrm{cm}^{2}$ & $98 \pm 3.64$ & $94.92 \pm 1.11$ & $94.23 \pm 0.84$ & 0.562 & $96.31 \pm 1.4$ & $93.96 \pm 0.95$ & $94.41 \pm 1.18$ & 0.362 \\
\hline Carcass weight, $\mathrm{kg}$ & $465.18 \pm 17.2$ & $456.29 \pm 5.27$ & $464.69 \pm 3.99$ & 0.428 & $459.64 \pm 6.65$ & $464.92 \pm 4.5$ & $461.64 \pm 5.63$ & 0.776 \\
\hline Meat ratio* & $65.17 \pm 1.23$ & $64.58 \pm 0.38$ & $63.50 \pm 0.29$ & 0.04 & $65.37 \pm 0.47^{\mathrm{a}}$ & $63.44 \pm 0.32^{\mathrm{b}}$ & $63.68 \pm 0.4^{\mathrm{b}}$ & 0.002 \\
\hline
\end{tabular}

a, $\mathrm{b}$ Means with the same superscript in the same row for each quality are not significantly different $(P<0.05) .{ }^{*}$ Meat ratio $=68.184-[0.625 \times$ back-fat thickness $(\mathrm{mm})]+[0.13 \times$ rib-eye area $\left.\left(\mathrm{cm}^{2}\right)\right]-[0.024$ carcass weight $(\mathrm{kg})]$. The Korean native cattle meat ratio has an additional 3.23 added after calculation.

nation genotypes of CC-CC, CT-CC, and TT-CC had a low least square mean for back-fat thickness compared to the steer group with other combinations. On the other hand, the steer group with the combination genotype had the highest least square mean for the meat ratio.

$H S P B 1$ is expressed in many vertebrate tissues, particularly muscle. In a previous study, Zhang et al. (2012) demonstrated that the HSPBI expression level was higher in the skeletal muscle of bulls than that of steers. Thus, in this study, the steer group in Korean cattle was used. As shown in Tables 1 and 2, although there were no significant differences between these SNPs and the rib-eye area, the mean in the group with the CC genotype of g.2352 T >C SNP and combi- nation genotypes of CC-CC, CT-CC, and TT-CC were found to be numerically higher than that in other groups.

The cellular development associated with adipose tissue growth involves both cellular hypertrophy (increase in size) and hyperplasia (increase in number). Rajesh et al. (2010) reported that $H S P B I$ interact with insulin-like growth factor receptor 1 and its signal transducer, the serine/threonine kinase Akt, which together modulate an adipocyte metabolism. Specifically, the previous results suggested that $H S P B 1$ was negatively correlated with an adipose metabolism (Kim et al., 2011). Therefore, our results regarding the least square mean of back-fat thickness and the rib-eye area were found to be similar. 
Table 2. The multiple effect of genotype combination of pairwise SNPs on carcass traits in commercial Korean native steer population.

\begin{tabular}{|c|c|c|c|c|c|c|c|}
\hline \multirow{2}{*}{ Carcass trait } & \multicolumn{6}{|c|}{ Genotype (number of animals) } & \multirow{2}{*}{$p$ value } \\
\hline & $\mathrm{CC}-\mathrm{CC}(6)$ & CT-CC (21) & CT-CT (45) & TT-CC (15) & TT-CT (46) & TT-TT (57) & \\
\hline Back-fat thickness, mm & $12.49 \pm 1.61^{\mathrm{a}}$ & $12.22 \pm 0.87^{\mathrm{a}}$ & $13.54 \pm 0.59^{\mathrm{ab}}$ & $11.73 \pm 1.04^{\mathrm{a}}$ & $15.39 \pm 0.59^{\mathrm{b}}$ & $14.32 \pm 0.53^{\mathrm{ab}}$ & 0.007 \\
\hline Rib-eye area, $\mathrm{cm}^{2}$ & $98 \pm 3.65$ & $96.82 \pm 1.96$ & $94.04 \pm 1.35$ & $94.84 \pm 2.36$ & $93.85 \pm 1.33$ & $94.4 \pm 1.19$ & 0.745 \\
\hline Carcass weight, $\mathrm{kg}$ & $465.18 \pm 17.2$ & $447.49 \pm 9.25$ & $461.55 \pm 6.35$ & $472.07 \pm 11.1$ & $467.43 \pm 6.28$ & $461.76 \pm 5.65$ & 0.521 \\
\hline Meat ratio* & $65.17 \pm 1.2^{\mathrm{a}}$ & $65.62 \pm 0.65^{\mathrm{a}}$ & $64.12 \pm 0.45^{\mathrm{ab}}$ & $65.08 \pm 0.78^{\mathrm{ab}}$ & $62.77 \pm 0.44^{b}$ & $63.67 \pm 0.39^{\mathrm{ab}}$ & 0.004 \\
\hline
\end{tabular}

a, b Means with the same superscript in the same row for each quality are not significantly different $(P<0.05) .{ }^{*}$ Meat ratio $=68.184-[0.625 \times$ back-fat thickness $($ mm $)]+[0.13 \times$ rib-eye area $\left.\left(\mathrm{cm}^{2}\right)\right]-[0.024$ carcass weight $(\mathrm{kg})]$. The Korean native cattle meat ratio has an additional 3.23 added after calculation.

In a previous study, it was reported that g.111. T > C SNP (rs208395876) was functional. In particular, our results identified that g.2352 T >C SNP was located in the acceptor site of the RNA splicing region.

Therefore, our results suggested that g. $111 \mathrm{~T}>\mathrm{C} \mathrm{SNP}$ and g.2352 T >C SNP could be causal mutations related to an adipose metabolism in Korean cattle steer.

\section{Conclusions}

We discovered three SNPs, including g.111 T>C, g.2352, and g.2548, which are respectively located in $5^{\prime}$ UTR, intron 2 , and $3^{\prime}$ UTR of the HSPB1 protein. Animals with a CC genotype of g.111 T > C SNP had a significantly higher meat ratio, and animals with a CC genotype of g. $2352 \mathrm{~T}>\mathrm{C}$ SNP had a significantly higher meat ratio and lower backfat thickness than those of other genotypes. Moreover, for the combination of g.111 T >C and g.2352 T >C SNPs, the CC-CC, CT-CC, and TT-CC genotypes' back-fat thicknesses were found to decrease while the meat ratios increased. In particular, a g.2352 T>C SNP was found to be located in the acceptor site of the RNA splicing site. Therefore, our results indicate that it could be a causal mutation related to an adipose metabolism in Korean cattle steer. 


\section{Appendix A}

Table A1. Primer information used in this study.

\begin{tabular}{|c|c|c|c|c|c|c|c|}
\hline SNP & dbSNP no. & & Primer sequence & $\mathrm{Tm}$ & Product & Prim & r sequence for Fluidigm SNP genotyping* \\
\hline g. $111 \mathrm{~T}>\mathrm{C}$ & rs 208395876 & $\begin{array}{l}\mathrm{F} \\
\mathrm{R}\end{array}$ & $\begin{array}{l}\text { AAGGTTCCAGATGTGGGCAG } \\
\text { ACCAGGGGTTGGGCGAAGAG }\end{array}$ & 62 & 781 & $\begin{array}{l}\text { ASP1 } \\
\text { ASP2 } \\
\text { LSP } \\
\text { STA }\end{array}$ & $\begin{array}{l}\text { CGCCCGCCACTTCTCC } \\
\text { CGCCCGCCACTTCTCT } \\
\text { GGCCATGCTGGCTGGTC } \\
\text { CCATAAAAGCGCTGCGGG }\end{array}$ \\
\hline g. $2352 \mathrm{~T}>\mathrm{C}$ & rs110832311 & $\begin{array}{l}\mathrm{F} \\
\mathrm{R}\end{array}$ & $\begin{array}{l}\text { CAGTCTCGGGCACCCAGATC } \\
\text { AGTGACGGATGGCACTGCAC }\end{array}$ & 60 & 783 & $\begin{array}{l}\text { ASP1 } \\
\text { ASP2 } \\
\text { LSP } \\
\text { STA }\end{array}$ & $\begin{array}{l}\text { CTACCCTCTTTGCCCGTCT } \\
\text { ACCCTCTTTGCCCGTCC } \\
\text { GGGTGGGGTCCACACCG } \\
\text { GACGCCCTTGTGTGTAACT }\end{array}$ \\
\hline g. $2548 \mathrm{C}>\mathrm{G}$ & rs 483014585 & $\begin{array}{l}\mathrm{F} \\
\mathrm{R}\end{array}$ & $\begin{array}{l}\text { CAGTCTCGGGCACCCAGATC } \\
\text { AGTGACGGATGGCACTGCAC }\end{array}$ & 60 & 783 & $\begin{array}{l}\text { ASP1 } \\
\text { ASP2 } \\
\text { LSP } \\
\text { STA }\end{array}$ & $\begin{array}{l}\text { AACAGCCGGAAAACAAGTAAAGAC } \\
\text { GAACAGCCGGAAAACAAGTAAAGAG } \\
\text { TGGGCCGCTGGGCTAA } \\
\text { CCCCGAAGCCGGGAAG }\end{array}$ \\
\hline
\end{tabular}

\footnotetext{
* ASP1: allele specific primer 1, ASP2: allele specific primer 2, LSP: locus specific primer, STA: specific target amplification.
} 
Data availability. The original data of the paper are available from the corresponding author upon request.

Author contributions. JKS performed the research. JKS and YL performed the data analyses and wrote the manuscript. JSL and HK revised the manuscript. YL and HGL designed the experiment. All authors reviewed and approved the final paper.

Competing interests. The authors declare that they have no conflict of interest.

Acknowledgements. This work was supported by a grant from the Next-Generation BioGreen 21 Program (project no. PJ013322012019), Rural Development Administration, Republic of Korea.

Financial support. This research has been supported by the NextGeneration BioGreen 21 Program (grant no. PJ013322012019).

Review statement. This paper was edited by Steffen Maak and reviewed by Jaedon Oh and one anonymous referee.

\section{References}

Arrigo, A.-P.: Mammalian HspB1 (Hsp27) is a molecular sensor linked to the physiology and environment of the cell, Cell Stress Chaperon., 22, 517-529, 2017.

Charge, S. B. and Rudnicki, M. A.: Cellular and molecular regulation of muscle regeneration, Physiol. Rev., 84, 209-238, 2004.

Dubińska-Magiera, M., Jabłońska, J., Saczko, J., Kulbacka, J., Jagla, T., and Daczewska, M.: Contribution of small heat shock proteins to muscle development and function, FEBS Lett., 588, 517-530, 2014.

Hamelin, M., Sayd, T., Chambon, C., Bouix, J., Bibé, B., Milenkovic, D., Levéziel, H., Georges, M., Clop, A., and Marinova, P.: Proteomic analysis of ovine muscle hypertrophy, J. Animal Sci., 84, 3266-3276, 2006.

He, H., Chen, S., Liang, W., and Liu, X.: Genome-wide proteomics analysis on longissimus muscles in Qinchuan beef cattle, Anim. Genet., 48, 131-140, 2017.

Jung, U., Kim, M., Wang, T., Lee, J., Jeon, S., Jo, N., Kim, W., Baik, M., and Lee, H.: Upregulated heat shock protein beta-1 associated with caloric restriction and high feed efficiency in longissimus dorsi muscle of steer, Livest. Sci., 202, 109-114, 2017.
Kim, N.-K., Lim, D., Lee, S.-H., Cho, Y.-M., Park, E.-W., Lee, C.S., Shin, B.-S., Kim, T.-H., and Yoon, D.: Heat shock protein B1 and its regulator genes are negatively correlated with intramuscular fat content in the Longissimus Thoracis muscle of Hanwoo (Korean cattle) steers, J. Agr. Food Chem., 59, 5657-5664, 2011.

Kim, Y.-S., Lee, J.-S., Lee, Y., Kim, W.-S., Peng, D.-Q., Bae, M.H., Jo, Y.-H., Baik, M., and Lee, H.-G.: Effect of glutamine on heat-shock protein beta $1(H S P B 1)$ expression during myogenic differentiation in bovine embryonic fibroblast cells, Food Sci. Biotechnol., 27, 829-835, 2018.

Lee, Y.-S., Oh, D., Kim, J.-J., Lee, J.-H., Park, H.-S., and Yeo, J.-S.: A single nucleotide polymorphism in LOC534614 as an unknown gene associated with body weight and cold carcass weight in Hanwoo (Korean cattle), Asian-Austr. J. Animal Sci., 23, 1543-1551, 2010.

Oh, D.-y., Nam, I., Hwang, S., Kong, H., Lee, H., Ha, J., Baik, M., Oh, M. H., Kim, S., and Han, K.: In vivo evidence on the functional variation within fatty acid synthase gene associated with lipid metabolism in bovine longissimus dorsi muscle tissue, Genes Genom., 40, 289-294, 2018.

Perng, M. D., Cairns, L., van den IJssel, P., Prescott, A., Hutcheson, A. M., and Quinlan, R. A.: Intermediate filament interactions can be altered by HSP27 and alphaB-crystallin, J. Cell Sci., 112, 2099-2112, 1999.

Rajesh, R. V., Kim, S.-K., Park, M.-R., Nam, J.-S., Kim, N.-K., Kwon, S., Yoon, D., Kim, T.-H., and Lee, H.-J.: Proteomic Functional Characterization of Bovine Stromal Vascular Cells from Omental, Subcutaneous and Intramuscular Adipose Depots, Asian Austral. J. Anim., 24, 110-124, 2010.

Sugiyama, Y., Suzuki, A., Kishikawa, M., Akutsu, R., Hirose, T., Waye, M. M., Tsui, S. K., Yoshida, S., and Ohno, S.: Muscle develops a specific form of small heat shock protein complex composed of MKBP/HSPB2 and HSPB3 during myogenic differentiation, J. Biol. Chem., 275, 1095-1104, 2000.

Sweetman, D.: The myogenic regulatory factors: critical determinants of muscle identity in development, growth and regeneration, Intech, 31-39, 2012.

Tucker, N. R. and Shelden, E. A.: Hsp27 associates with the titin filament system in heat-shocked zebrafish cardiomyocytes, Exp. Cell Res., 315, 3176-3186, 2009.

Zhang, Q., Lee, H.-G., Han, J.-A., Kang, S. K., Lee, N. K., Baik, M., and Choi, Y.-J.: Differentially expressed proteins associated with myogenesis and adipogenesis in skeletal muscle and adipose tissue between bulls and steers, Mol. Biol. Rep., 39, 953-960, 2012.

Zhang, Q., Lee, H.-G., Kang, S. K., Baik, M., and Choi, Y.-J.: Heatshock protein beta 1 regulates androgen-mediated bovine myogenesis, Biotechnol. Lett., 36, 1225-1231, 2014. 